\title{
Gait Generation and Control of a Hexapod Walking Robot
}

\author{
Yu Ya-xin ${ }^{1, *}$ and Jin $\mathrm{Bo}^{2}$ \\ ${ }^{I}$ Faculty of Mechanical Engineering and Automation, Zhejiang Sci-Tech University, Hangzhou 310018, China \\ ${ }^{2}$ The State Key Laboratory of Fluid Power Transmission and Control, Zhejiang University, Hangzhou 310027, China
}

\begin{abstract}
In this paper, the mechanical structure of a hexapod walking robot is presented and the kinematic model is established. The foot trajectory of each leg, which is optimized by genetic algorithms to minimize energetic cost, was proposed with two different gait patterns applied. Control system was divided into coordination-layer and execution-layer. Coordination-layer, was a PC responsible, for whole system's regulation. It could calculate each joint's expected trajectory according to user's definitions, and then transmitted object position data to execution-layer through serial port. The execution-layer used LPC2132 based on ARM7 for core controller. Control system realized the human-machine interaction, gait generation, complex kinematic calculation and servomotor control. Experimental results of the robot shows that it could walk smoothly without obvious body undulation.
\end{abstract}

Keywords: Control system, hexapod walking robot, joint trajectory planning, kinematics.

\section{INTRODUCTION}

Multi-legged walking robot generally includes quadruped walking robot, hexapod walking robot, and eight-legged walking robot [1-3]. In recent years, multi-legged walking robot is broadly recognized for superior performance in its potential to be an effective and efficient transportation device on irregular or difficult terrains. The possible application areas for legged robots include the explorations of volcanoes [4], forest [5-7], humanitarian demining [8-12] and so on. Most of these circumstances usually go along with hazardous and unstructured, complex environments, which cannot be entered by humans. Compared with wheeled or tracked locomotion, legged robot has variety of advantages on coping with these scenarios although it is quite slow as well as its payload weight-to-own-weight ratio is not good enough until now [13]. On the one hand, the contact points between the wheeled or tracked robots and ground surface is continuous while those between the multi-legged robots and ground are discrete. Considering that due to obstructed paths, the appropriate continuous contact trajectories could not be easily found in the unstructured terrain areas. On the other hand, the body trajectories of legged robot would not be affected by uneven ground because of the agility and flexibility of multi degrees of freedom (DOFs) leg mechanisms. Although the multi-legged robot can only be achieved by a great number of joints, all of which have to be controlled by a complex control system, the advantages still attract the researchers. Hence, various hexapod walking robots, such as the famous ASV [14, 15], the JQuadRobot $[16,17]$ and so on, have been developed in the past 30 years.
Based on imitating the walking feature of mammals, this paper presents the design concept and hardware configuration of a hexapod walking robot, while especially illustrates the gait generation, joint trajectory planning method, and the hardware \& software of the control system.

\section{STRUCTURE DESIGN AND GAIT PATTERN}

The robot designed in this work consists of two parts as shown in Fig. (1): one main body and six leg mechanisms. The main body is a constructive aluminum frame to carry both onboard controller and the power supply. The leg mechanisms consist of two links which are connected mutually by a knee joint. Each leg is attached to the main body by the hip joints. The robot acquires 12 degrees of freedom (DOF) by the high-torque-output rudder servo at each joint. The motions of the robot are driven by the rotation of 12 rudder servos which are coordinated by a controller. Every joint rotates according to the planned trajectory and thus the robot can move forward or backward, traverse obstacles, etc. Rubber pads are set to the foot ends of legs to reduce landing impacts and avoid slippage [18].

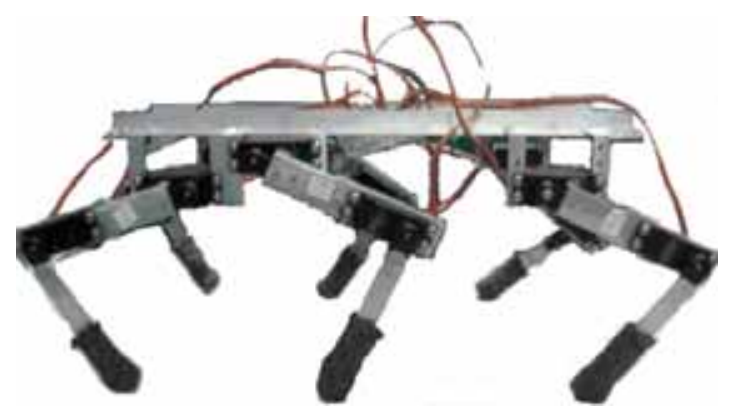

Fig. (1). General structure of the robot. 


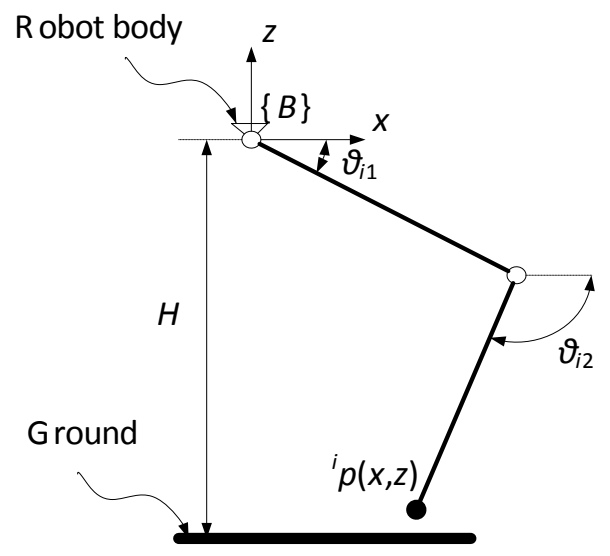

Fig. (2). Single-leg kinematic model.

In this study we assumed that the body walks along a straight path on horizontal ground at a constant velocity $\mathrm{V}$ with a constant height $\mathrm{H}$. The center of gravity (COG) is located at the geometric center of the vehicle body. The reference frame is defined as a body-fixed frame, having its origin at the COG. As a statically stable hexapod robot, the alternating tripod gait and the wave gait are both adopted in our study. For this hexapod robot model, the alternative tripod gait can be expressed in the gait formula as:

$\varphi_{1}=\varphi_{3}=\varphi_{5}=0$

$\varphi_{2}=\varphi_{4}=\varphi_{6}=1 / 2$

Where, all legs are numbered in a counterclockwise direction. $0 \leq \varphi_{i}<1$ represent the different phases of the legs motion between leg-i and the left-front leg(leg-1).

The phase modified wave gait, which is proved to be more efficient than the conventional wave gaits by Erden and Leblebicioglu [19], can be expressed in the gait formula as:

$$
\begin{array}{ll}
\varphi_{1}=0 & \varphi_{6}=\operatorname{Frac}\left[\frac{1}{2}\right], \\
\varphi_{2}=\left\{\begin{array}{ll}
\operatorname{Frac}[-\beta], & \beta \leq \frac{2}{3}, \\
\operatorname{Frac}\left[\frac{1}{3}\right], & \beta>\frac{2}{3},
\end{array} \quad \varphi_{5}= \begin{cases}\operatorname{Frac}\left[\frac{1}{2}-\beta\right], & \beta \leq \frac{2}{3}, \\
\operatorname{Frac}\left[\frac{51}{6}\right], & \beta>\frac{2}{3},\end{cases} \right. \\
\varphi_{3}= \begin{cases}\operatorname{Frac}[-2 \beta], & \beta \leq \frac{2}{3}, \\
\operatorname{Frac}\left[\frac{2}{3}\right], & \beta>\frac{2}{3},\end{cases}
\end{array}
$$

Where, $\beta$ is the duty factor and is defined as the fraction of the cycle time $\mathrm{T}$ when a leg remains on the ground.

The fractional function Frac $[\mathrm{x}]$ of a real number $\mathrm{x}$ is defined as follows:

$$
\begin{aligned}
& \operatorname{Frac}[x]= \begin{cases}\text { the fraction part of } x, & x \geq 0 \\
1-(\text { the fraction part of }\{-x\}), & x<0\end{cases}
\end{aligned}
$$

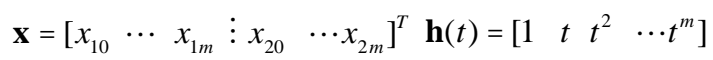

\section{KINEMATICS AND FOOT TRAJECTORY PLAN- NING}

The inverse kinematic model of single leg is defined as Fig. (2). The determination of the joint positions and velocities is the inverse problem of the kinematics, that is, the position vectors ${ }^{i} \tilde{P}=\left[\begin{array}{lll}{ }^{i} p_{x} & { }^{i} p_{y} & { }^{i} p_{z}\end{array}\right]^{T}$ in the body-fixed frame $\{\mathrm{B}\}$ are known from the desired walking gait, but the joint actuation variables $\theta_{i 1}$ and $\theta_{i 2}$ are to be found. Note that if the leg is a supporting leg, ${ }^{i} P$ is unchangeable. Otherwise, at arbitrary moment, it is varying along with the foot trajectory of the swing leg. According to this model, the joint angles are determined with the relations Eq. 3 .

$$
\begin{aligned}
& \theta_{i 1}=-2 \arctan \left(\frac{a_{i 1}-\sqrt{a_{i 1}^{2}+a_{i 2}^{2}-a_{i 3}^{2}}}{a_{i 2}+a_{i 3}}\right) \\
& \theta_{i 2}=-\arccos \left(\frac{{ }^{i} p_{z}{ }^{2}+{ }^{i} p_{x}{ }^{2}-L_{1}^{2}-L_{2}^{2}}{2 L_{1} L_{2}}\right) \\
& \text { Where, } \quad a_{i 1}=2 L_{1}{ }^{i} p_{z}, \quad a_{i 2}=2 L_{2}{ }^{i} p_{x}
\end{aligned}
$$
$a_{i 3}={ }^{i} p_{x}{ }^{2}+{ }^{i} p_{z}{ }^{2}+L_{1}^{2}-L_{2}^{2}, \mathrm{~L} 1, \mathrm{~L} 2$ are the lengths of thigh and shank links.

During the stance phase, the relative velocity of the foot to the body is given by:

$$
{ }^{i} \dot{\tilde{P}}=\left[\begin{array}{lll}
-\frac{S}{\beta T} & 0 & 0
\end{array}\right]^{T}
$$

Where, $\mathrm{S}$ is the stride length.

During the transfer phase, in order to make a single smooth motion of the foot, some constraints are needed to satisfy the following requirements [20].

Trajectories are smooth so that acceleration of legs is easier for the motor controllers and foot motions would not give undesirable reaction to the body.

(1) Trajectories must be smooth, hence the motor controllers can easily control the acceleration of legs, and foot motions would not be harmful to the body.

(2) The vertical portion of trajectories should be enough while landing and leaving to avoid the feet's horizontal slippage.

(3) The landing motion of feet should be decelerated in order to avoid landing impacts.

(4) Trajectories should have enough vertical clearance during the transfer phase in order to avoid the obstacles.

Therefore, a trajectory generation technique using polynomial functions is used to determine the angles and angular velocities of both hip and knee joints as Eq. (5).

$$
\mathbf{q}(t)=\left[\begin{array}{ll}
\theta_{1}(t) & \theta_{2}(\mathrm{t})
\end{array}\right]^{T}=\left[\begin{array}{cc}
\mathbf{h}(t) & 0 \\
0 & \mathbf{h}(t)
\end{array}\right] \mathbf{x}
$$

Where, 


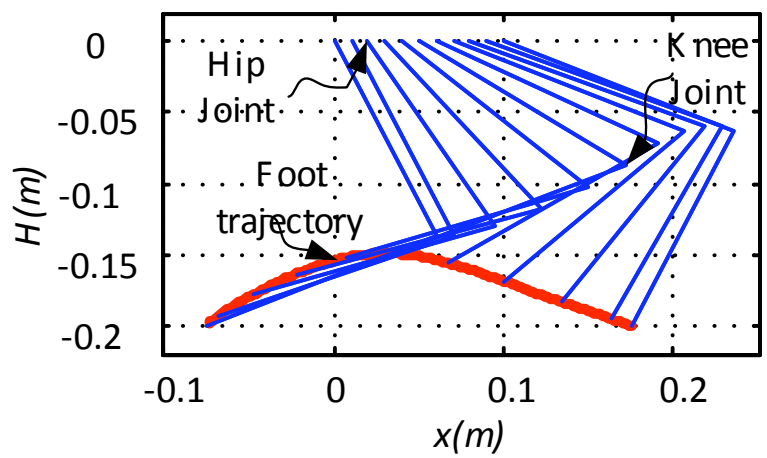

Fig. (3). The optimal foot trajectory in transfer phase.

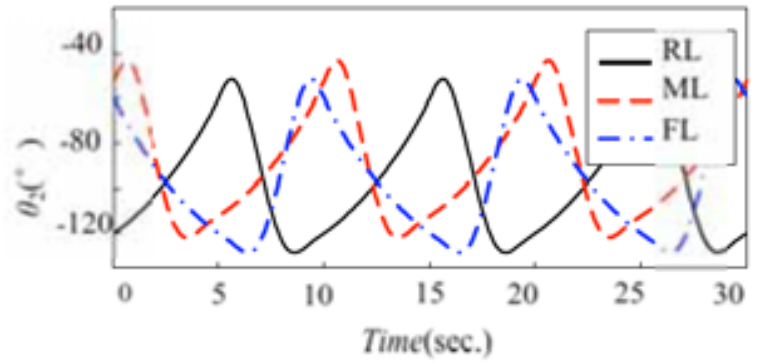

(a) the knee joint

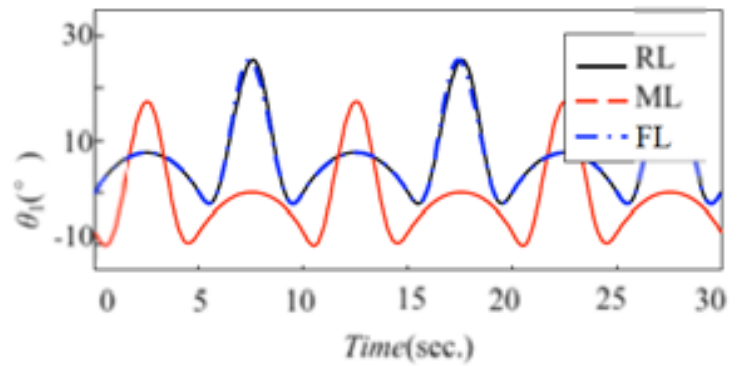

(b) the hip joint

Fig. (4). The simulation result of joint angular trajectories.

$\mathrm{M}$, is the polynomial degree, which is decided by the constraints number, $\mathrm{x}$ is the undetermined coefficient.

To optimize the energetic cost during each leg's transfer phase, the objective function could be written as:

$$
\begin{aligned}
& f(x)=\min _{x}\left(\int_{0}^{T} \sum_{i=1}^{2} P_{i}(t) d t\right) \\
& =\min _{x}\left(\int_{0}^{T}\left(\|\Gamma(\mathbf{q}, \dot{\mathbf{q}}, \ddot{\mathbf{q}})\|^{2}+\max (\Gamma(\mathbf{q}, \dot{\mathbf{q}}, \ddot{\mathbf{q}}), 0)\right) d t\right)
\end{aligned}
$$

Where

$$
\Gamma(\mathbf{q}, \dot{\mathbf{q}}, \ddot{\mathbf{q}})=\mathbf{D}(\mathbf{q}) \ddot{\mathbf{q}}+\mathbf{H}(\mathbf{q}, \dot{\mathbf{q}})+\mathbf{C}(\mathbf{q})=\left[\tau_{1}(t) \tau_{2}(t)\right]^{T}
$$

$P_{i}(t)$ is joint i's instantaneous power, $\tau_{i}(t)$ is the joint i's torque; $D$ is the acceleration-related symmetric matrix, which involves the inertia tensor; $H$ is the Coriollis and centrifugal matrix and $C$ is the gravity matrix.

In this study, genetic algorithms have been used to search the optimal trajectory of each foot. Fig. (3) is the optimiza- tion results of single-leg's foot trajectory with the stride length $S=0.15 \mathrm{~m}$, the body height $H=0.2 \mathrm{~m}$ and the clearance $\mathrm{h}=0.05 \mathrm{~m}$. The left legs' joint angular trajectories during three walking cycle time which are solved by Eq. 3 were presented in Fig. (4). Here, RL means "rear left leg"; ML means "middle left leg"; FL means "front left leg".

\section{CONTROL SYSTEM DESIGN}

The control system consists of two layers with different functions. The coordination layer is a host computer whose responsibility includes trajectory planning and transmitting position instructions of servos to the execution layer. The execution layer is a controller with a main chip of LPC2132 produced by PHILIPS based on ARM7TDMI-S core. This layer receives the control instructions from the coordination layer and drives the 12 rudder servos. The two layers communicate through $\mathrm{RS}-232$ serial ports.

The hardware configuration of the control system is shown in Fig. (5). The execution layer consists of the core controller module, serial communication module, power management module, motor driving module, etc. 


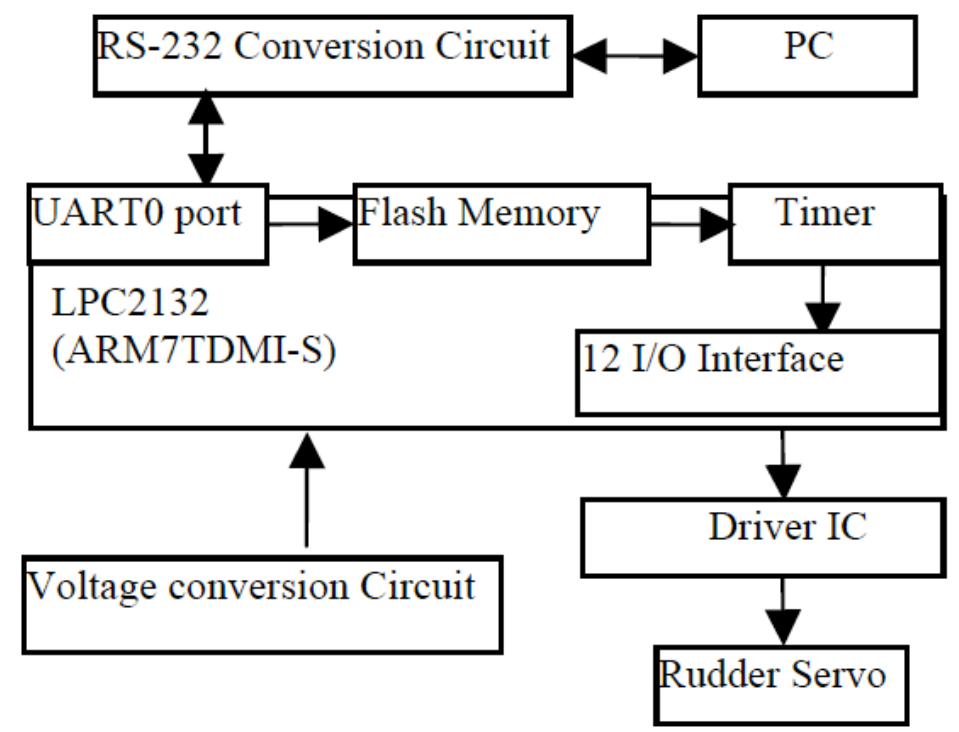

Fig. (5). Hardware configuration of the controller.

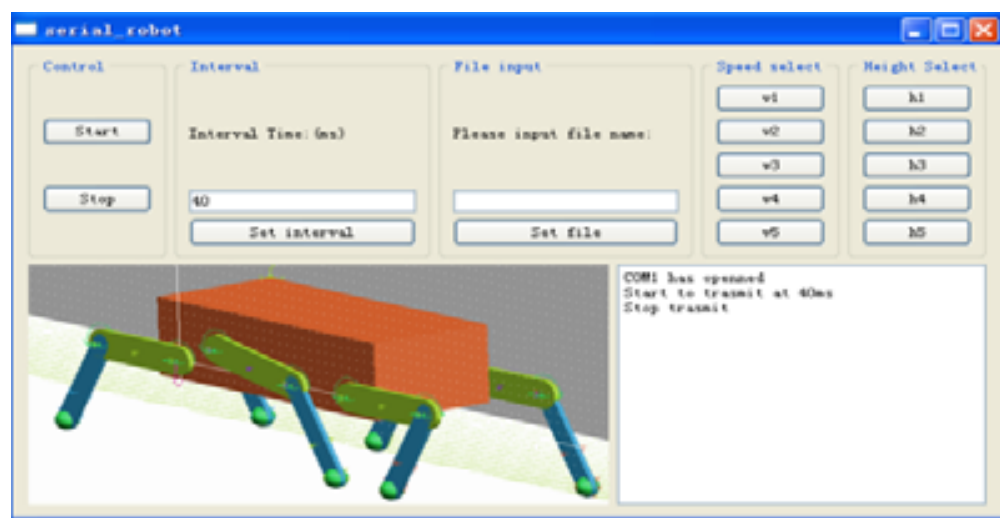

Fig. (6). GUI of the host computer.

The host computer adopted QT for interface development. The graphic user interface (GUI) based on QT is developed in Qtopia Core which offers serial communication function as well as characteristics of real-time and transplantation. The GUI of coordination layer is shown in Fig. (6). In this interface, several tasks can be completed as follows:

Set path, height and speed for walking;

Calculate the objective trajectory of 12 rudder servos;

Convert the continuous trajectory to pulse width time series for servo-motor control;

After starting the walking gait, set different intervals and transmit new data of objective position to execution layer through RS-232 port;

Start or stop the robot.

The execution layer has two main functions as follows:

Firstly, it receives pulse width sequences of objective position sent by the host computer at certain intervals trough RS-232 serial communication port for 12 rudder servos.

Secondly, it drives 12 rudder servos with PWM signals. In every period of $20 \mathrm{~ms}$, a high level signal lasting for $0.5 \mathrm{~ms} \sim 2.5 \mathrm{~ms}$ can drive a servo to rotate an angle of $0^{\circ} \sim 180^{\circ}$.
In this work, time slicing method is adopted to control 12 servos because of insufficient timer resource and small duty cycle. The maximum of pulse width is $2.5 \mathrm{~ms}$ so one timer can control 8 servos with 8 time slices in a period of $20 \mathrm{~ms}$. LPC2132 can configure three 32-bit timers at most so the degree of freedom (DOF) of the robot can be increased to 24 in the future. In this paper, suppose the servos of 1 12 are controlled by pulses of M1 M12 divided into three groups: M1 M4, M5 M8 and M9 M12 which are managed by interrupt service routines (ISR) for Timer0、Timer1 and PWM0, then the control sequences can be set as shown in Fig. (7).

Initially all signals sent to the motors are low level. Once a timer interrupt happens and interrupt service routine is called, the controller will have to decide which servo to allocate time slice and estimate the port state of that servo. If the state is low level, the port should be set to make the servo rotate. Meanwhile, transmit the pulse width value from corresponding register to the timer and restart it; If the state is high level which indicates that the servo has rotated to the objective position, clear the port and transmit the value of difference between $2.5 \mathrm{~ms}$ and servo pulse width value to the timer and restart it, finally, direct the time slice pointer to next servo. The 3 timers controlling different ports are simi- 


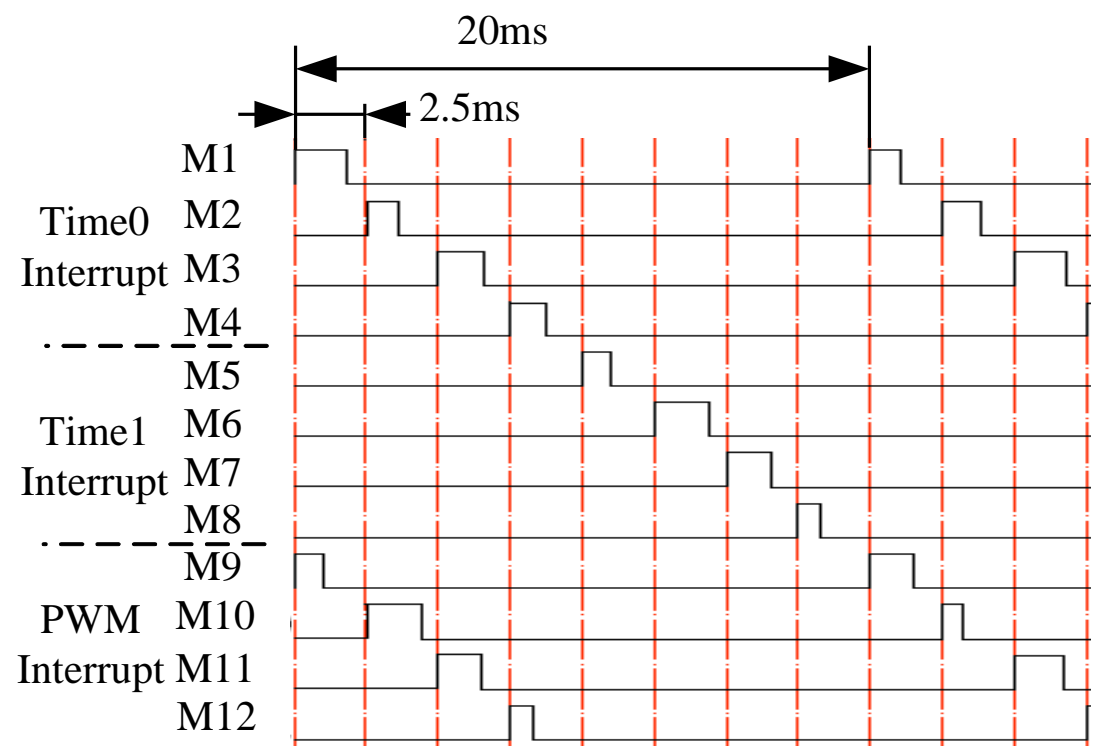

Fig. (7). Control sequence diagram for 12 rudder servos.

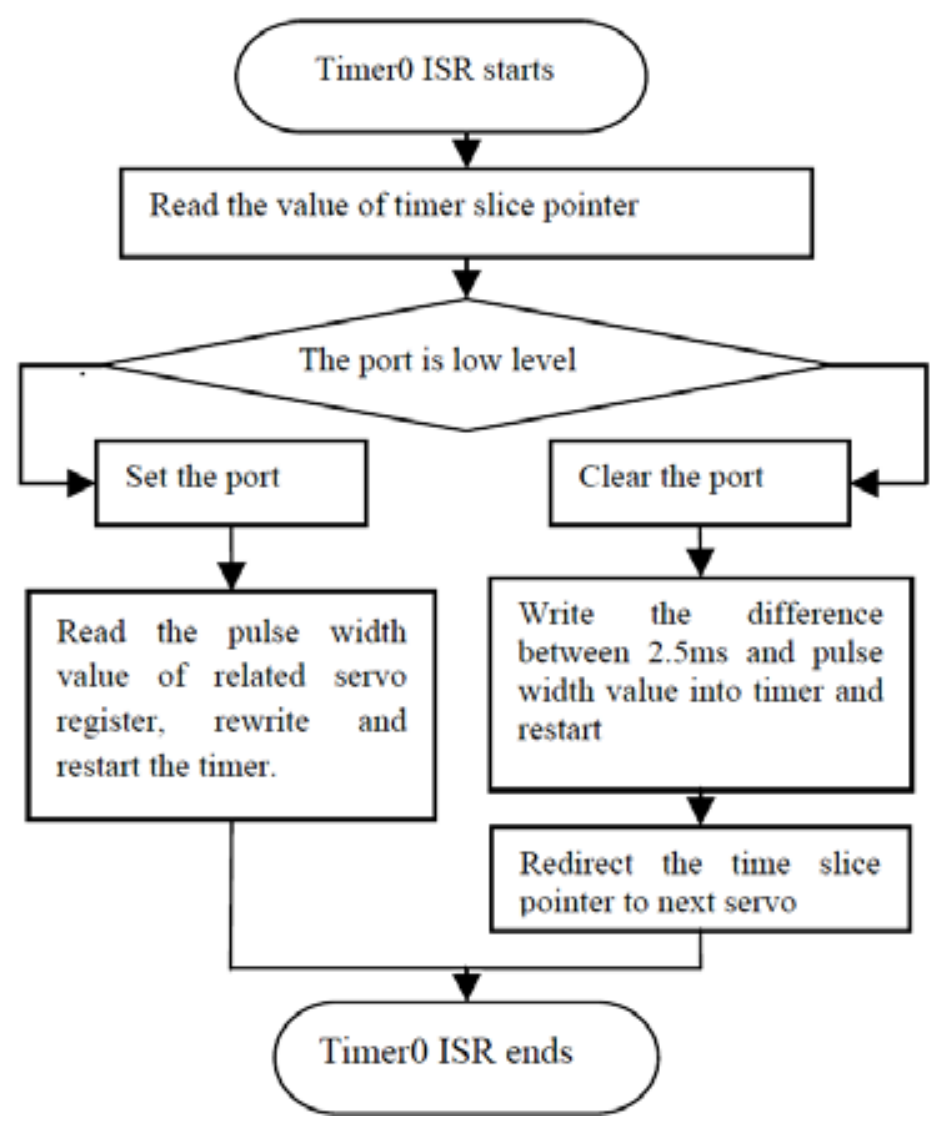

Fig. (8). Flow chart of Timer0 interrupt program.

lar in principle, but Timer 1 starts $10 \mathrm{~ms}$ later than Timer0 and PWM0 during every period. It should be noticed that the difference value between $12.5 \mathrm{~ms}$ and the pulse width of servo4 should be transmitted to Timer0 at the falling edge of M4 to trigger Timer0 again every period of $20 \mathrm{~ms}$ and redirect the time slice pointer to rudder servo1. The processing methods of falling edge for M8 and M12 are similar to that of M4.
The interrupt service routine of Timer0 is shown in Fig. (8) to demonstrate the process of time slicing for rudder servos.

Through time slicing method, the controller only has to handle 1 2 ISR for motors. Meanwhile, heavy tasks are avoided such as frequent ISR reply, stack operations, interrupt nesting, etc. 

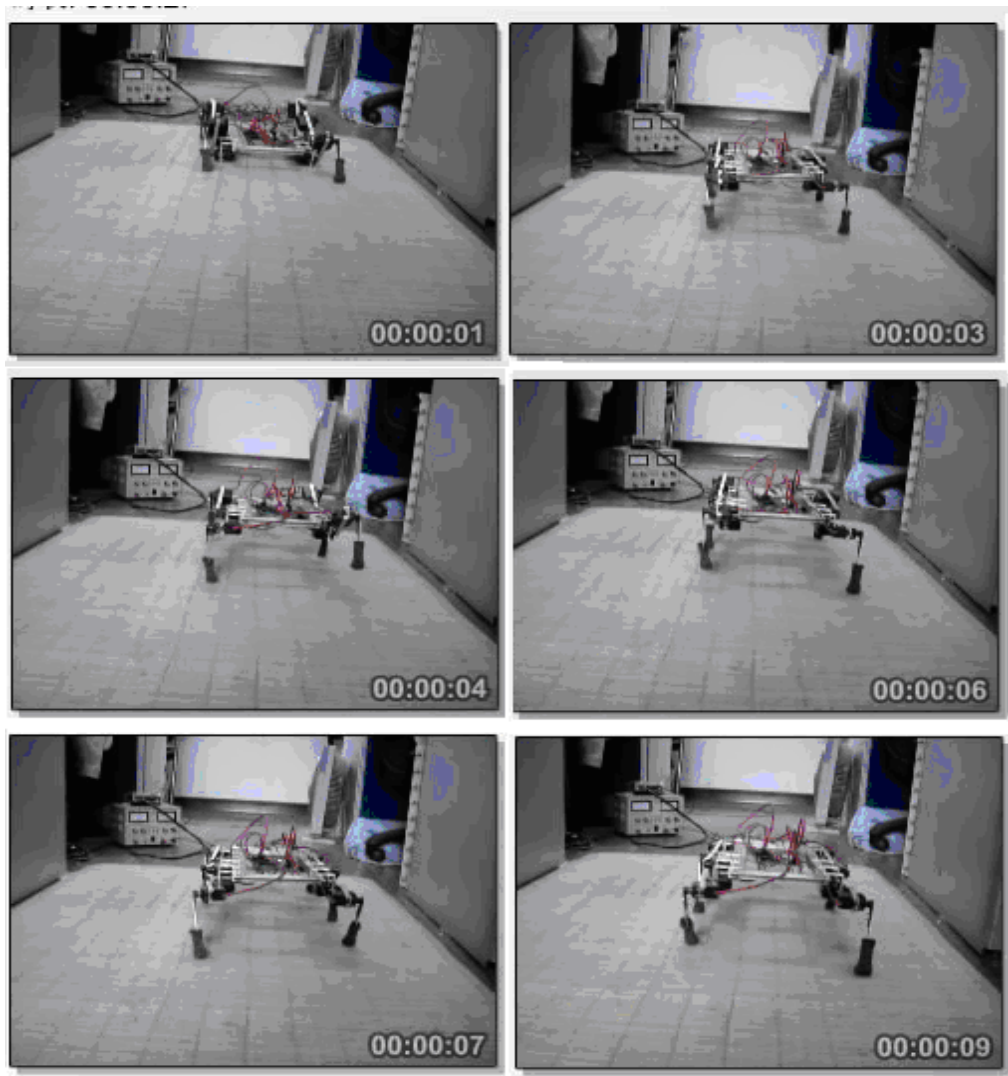

Fig. (9). The photos of walking experiments.

\section{EXPERIMENTAL RESULTS}

Based on the above principles and design scheme, a prototype of the robot is fabricated with length of $34 \mathrm{~cm}$, width of $24 \mathrm{~cm}$, weight of $1 \mathrm{~kg}$ and controllable height of $8 \sim 20 \mathrm{~cm}$ for walking.

Experimental results of the prototype show that the robot can walk smoothly without obvious body undulation, as shown in Fig. (9). It begins to wobble slightly without slipping when walking at a speed of $5 \mathrm{~cm} / \mathrm{s}$. When the speed reaches $10 \mathrm{~cm} / \mathrm{s}$, phenomenon of wobbling and slipping become slightly aggravated. The linear deviation is $4 \mathrm{~cm}$ when the distance of marching forward is $3 \mathrm{~m}$.

\section{CONCLUSION}

This paper addresses the development of a hexapod walking platform with the gait generation and trajectory planning method proposed. The control system of the hexapod walking robot presented in this paper can manipulate the robot to walk with different speeds or heights in various paths. The experiments show that the robot performed satisfying characteristics of balance, stability and rapidity while the control system can obtain excellent control effects.

\section{CURRENT AND FUTURE DEVELOPMENTS}

With the structure and method mentioned above, the hexapod robot can walk straight with different speed and body height on a plain ground. The future work will install a third

degree for each of the leg, with that, the robot can turn around to any direction freely. A gyroscope will be installed on the body to control the posture of the robot, and force sensor will be installed on each foot, hence the robot has the ability to walk on rough terrain.

\section{CONFLICT OF INTEREST}

The authors confirm that this article content has no conflict of interest.

\section{ACKNOWLEDGEMENTS}

This work was supported by Zhejiang Sci-Tech University 521 talent cultivation program and Zhejiang provincial Natural Science Foundation of China (No.LY12E05028).

\section{REFERENCES}

[1] Kobayashi, Sonehara, Tanaka, Azumi, and Ogawa, "Four-Leg Mobile Robot", J. P. Patent 2005046950, July 28, 2003.

[2] C. H. Yang, "Hexapod robot device", U. S. Patent 20120259461 A1, October 11, 2012.

[3] M. D. Summer, R. F. Varley, L. H. Davis, and J. A.Stiver, "Control system and related method for multi-limbed, multi-legged robot", U. S. Patent 7339340B2, March 4, 2008.

[4] J. E. Bares, and D. S. Wettergreen, "Dante II: Technical description, results and lesson learned", The International Journal of Robotics Research, vol. 18, no. 7, pp. 621-649, 1999.

[5] [Online] Walking Machine Catalogue, http://www.walkingmachines.org/, 2008.

[6] M. Paakkunainen, "Leg Mechanism”, U. S. Patent 6109378, August 29,2000 . 
[7] M. Paakkunainen, "Method for Adjusting Supply Pressure", U. S. Patent 6305163 B1, October 23, 2001.

[8] P. Gonzalez, J. A. Cobano, and E. Garcia, "A six legged robotbased system for humanitarian demining missions", Mechatronics, vol. 17, pp. 417-430, 2007.

[9] Y. Baudoin, and E. Colon, "Humanitarian demining and robotics", IEEE International Conference on Control Applications, Trieste, Italy, 1998.

[10] K. Nonami, Q. J. Huang, and D. Komizo, "Humanitarian mine detection six-legged walking robot", the $3^{\text {rd }}$ International Conference on Climbing and Walking Robots, 2000.

[11] Q. J. Huang, and K. Nonami, "Humanitarian mine detecting sixlegged walking robot and hybrid neuro walking control with position/force control", Mechatronics, vol. 13, pp. 773-790, 2003.

[12] P. Gonzalez, E. Garcia, and J. Estremera, "SILO6: Design and Configuration of a Legged Robot for Humanitarian Demining", International Advanced Robotics Program, International Workshop on Robots for Humanitarian Demining, Vienna, Austria, 2002.

[13] M. Nitulescu, and V. Stoian, "Modelling and control aspects of specific mobile robot", Mechanika, vol. 1, no. 69, pp. 54-58, 2008.
[14] K. J. Wardron, V. J. Vohunout, A. Pery, and R. B. Mcghee, "Configuration design of the adaptive suspension vehicle", The International Journal of Robotics Research, vol. 3, no. 2, pp. 37-48,1984.

[15] J. E. Bares, and W. William, "Orthogonal legged walking robot", U. S. Patent 5005658, April 9, 1991.

[16] S. Vatau, V. Ciupe, C. Moldovan, and I. Maniu, "Mechanical design and system control of quadruped robot", Mechanika, vol. .5, no. 85 , pp. 56-60,2010.

[17] H. Shigeo, O. Toshihito, F. Yasushi, D. Takahiro, and H. Ryuichi, "Four-Legged Walking Robot", JP Patent, 2006255816 (A), Sep. $28,2006$.

[18] Husha, "Research on Key Techniques on the Straight Walking of Hexapod Robot", Master Dissertation, Zhejiang University, Hangzhou, China 2008.

[19] M. S. Erden, and K. Leblebicioglu, "Analysis of wave gaits for energy efficiency", Auton Robot, vol. 23, pp. 213-230. 2007.

[20] L. Q. Wang, "Model Set-up of a Bionics Amphibious Crab-liked Robot and Walking Legs' Coordinate Control Technology Research", Ph.D. dissertation, Control Theory and Control Engineering, Harbin Engineering University, Harbin, China, 2003.

(C) Ya-xin and Bo; Licensee Bentham Open.

This is an open access article licensed under the terms of the Creative Commons Attribution Non-Commercial License (http://creativecommons.org/licenses/by-nc/3.0/) which permits unrestricted, non-commercial use, distribution and reproduction in any medium, provided the work is properly cited. 